\title{
MULTISCALE MODELING IN THERMAL MATERIALS PROCESSING
}

\author{
Yogesh Jaluria \\ Board of Governors Professor \& Distinguished Professor \\ Mechanical and Aerospace Engineering Department \\ Rutgers University, Piscataway, NJ, USA \\ Email: jaluria@jove.rutgers.edu
}

\begin{abstract}
An important aspect in most thermal systems involved in manufacturing and materials processing is that of modeling different length and time scales that arise and then coupling these to simulate the complete process. The changes in the material occur mainly at micro- and nanoscale levels, whereas the products being fabricated and the operating conditions are usually at the macro- or engineering scale. Depending on the dimensions, different transport regimes may arise, with different analysis and experimentation being needed at different scales. The overall modeling and simulation is then employed to determine the quality of the fabricated product, the effect on the environment, process efficiency and optimal conditions. This paper focuses on such materials processing applications where multiple length and time scales are of interest. It presents the modeling at the different scales and then addresses the question of coupling the different models to obtain the overall model for the system or process. Both numerical and experimental methods to obtain results at the small length scales are discussed.
\end{abstract}

Keywords: Multiscale, materials, modeling, thermal processing

\section{NOMENCLATURE}

$c_{p} \quad$ Specific heat at constant pressure

$E_{d}, E_{p} \quad$ Activation energies of defects and precursors

$K \quad$ Thermal conductivity; Boltzmann constant; reaction rate

$L \quad$ Optical fiber preform length

$n_{d}, n_{p} \quad$ Defect and precursor concentrations

$p \quad$ Pressure

$\mathrm{r} \quad$ Radial coordinate distance

$\mathrm{R} \quad$ Universal gas constant

$\mathrm{S}_{\mathrm{r}} \quad$ Radiation source term

$\mathrm{t} \quad$ Time

T Temperature

$\begin{array}{ll}\mathrm{u} & \text { Radial velocity } \\ \mathrm{v} & \text { Axial velocity } \\ \mathrm{z} & \text { Axial coordinate distance } \\ \mathrm{v} & \text { Kinematic viscosity } \\ v^{\prime} & \text { Constant in } E^{\prime} \text { defect equation } \\ \Phi & \text { Viscous dissipation term } \\ \rho & \text { Density }\end{array}$

Subscripts

melt Softening point of silica glass

\section{INTRODUCTION}

Multiscale modeling is critical in most manufacturing and materials processing systems, since the basic transformations in the material largely occur at micro/nanoscale levels, whereas the products and the overall system are at engineering scale [1]. However, since our interest lies in the product quality, and the cost and efficiency of the process, it is important to model the different scales accurately and couple these to obtain the overall model for the process.

Several important considerations arise in the modeling of multiple length scales. The first one concerns distinct regions where different scales apply. In the chemical vapour deposition (CVD) process, which is used to fabricate thin films, for instance, the thin film thickness varies from a few nanometers to a few microns [2, 3]. Modeling for the deposition involves these small length scales, whereas the bulk flow is modelled as macroscopic. The second aspect concerns the coupling of the results for the micro/nanoscale region with those for the bulk flow and heat transfer in the reactor. Similarly, the time scales involved in different components of a system may be quite different. Modelling of the system then involves considerations similar to those for multiple length scales. The third aspect concerns the link between transport processes at small scales to the changes in the material. In manufacturing processes such as crystal drawn from silicon melt, manufacture of optical glass fibers, surface coating, 
and gel formation in food extrusion, the material transformation, impurities and defects are difficult to model because of complicated mechanisms and lack of information on material behaviour. Experimentation at the small scales may then be used to provide inputs to the model. Finally, the product characteristics and properties need to be determined as functions of the operating conditions in order to vary the process to obtain desired products.

Figure 1 shows a few practical circumstances in materials processing where multiple length scales are of interest. The CVD and the crystal growing processes have already been mentioned. The polymer extrusion system is also shown. In all these cases, multiscale modeling is essential to the prediction, characterization, design and optimization of the process to obtain the desired product characteristics at minimal cost.

\section{- Vertical Reactor}

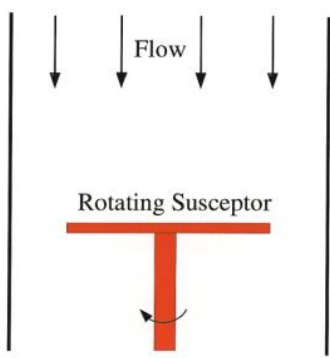

(a)

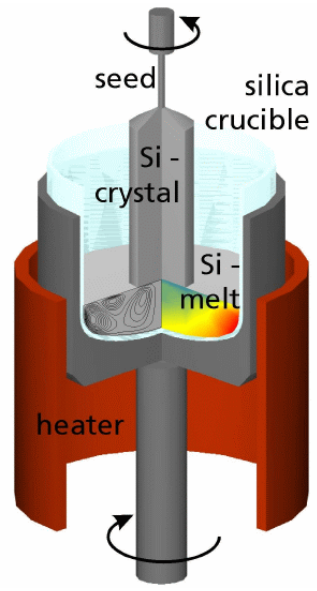

(b)

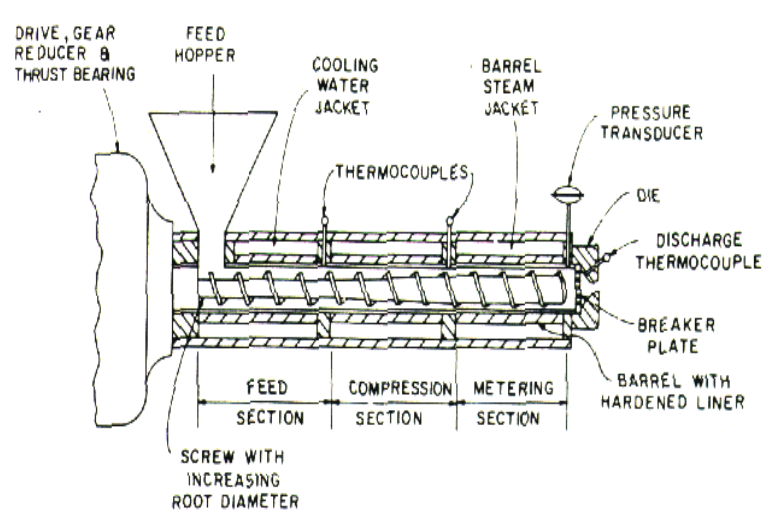

(c)

FIGURE 1. A few manufacturing processes in which multiple length scales are of interest: (a) Chemical vapor deposition; (b) Czochralski crystal growing; (c) Polymer screw extrusion.

\section{DIFFERENT CIRCUMSTANCES}

Two main circumstances are encountered when dealing with multiple scale in materials processing. These are:

1. Multiple scales in separate domains

2. Multiple scales within a given region

The former case is a common approximation and is easier to model, since each region can be treated separately. Different regions often involve different governing mechanisms and the solutions are then coupled at the boundaries. The second case is more complicated and applies in most material processing applications, where micro/nanoscale material changes occur within the macroscopic flow and thermal transport. The processes occurring over the small scales are determined experimentally or analytically and the results are then coupled with the bulk transport.

\section{MULTIPLE SCALES IN DIFFERENT DOMAINS}

As an example, let us consider the batch annealing system shown in Fig. 2(a) for the annealing of steel sheets that have been rolled up into coils. Three such stacked coils are shown, along with the other components of the furnace. The furnace is heated by the flow of flue gases from the blast furnace in the steel plant and an inert environment is maintained next to the steel coils.

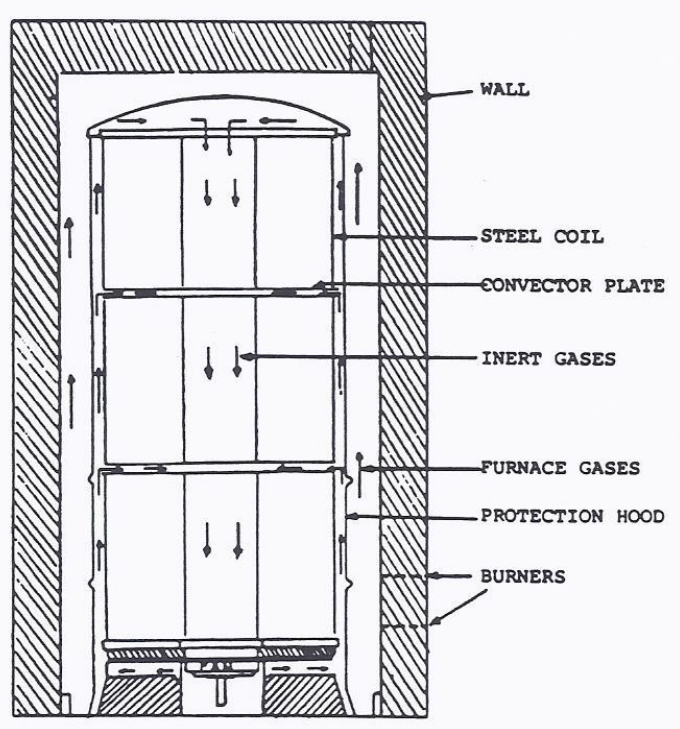

(a) 


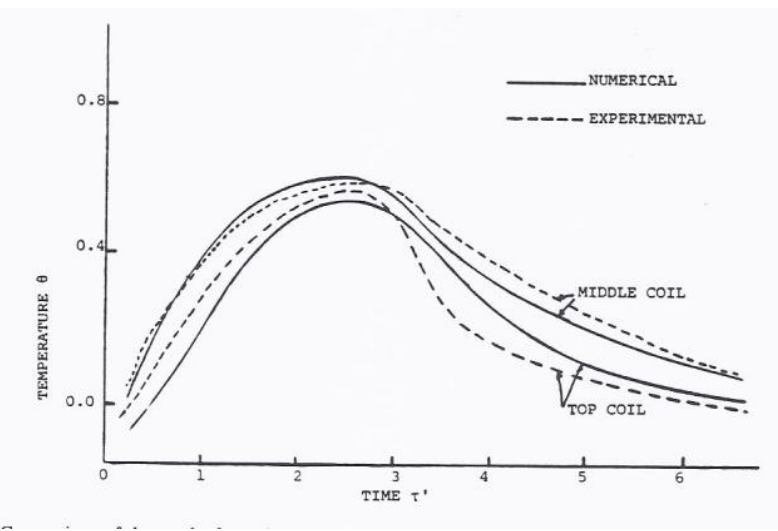

(b)

FIGURE 2. (a) A typical furnace for the batch annealing of coils of steel sheets; (b) Measured and calculated temperatures.

The annealing process involves heating up the material to be heat treated to the recrystallization temperature of around $723{ }^{\circ} \mathrm{C}$ and then cooling it gradually so that the residual stresses are relieved and the material ductility is improved [4]. Different components have different length and time scales. The overall furnace is $7-10 \mathrm{~m}$ in height, whereas several components are only millimetres in thickness. The walls heat up slowly, with large response times, whereas the gases respond within seconds. Thus, different length and time scales can be used to consider different regions and components as separate and modeled with their own governing mechanisms and equations. For instance, a quasi-steady behaviour may be assumed for the walls and lumped analysis used for smaller components such as the convector plate.

The preceding approach can be used to develop the model for the entire thermal process undergone by the steel coils as well as the system. The simulations in different regions or components are matched at the boundaries. Reduced complexity in different components may be used to simplify the model [5]. The numerical predictions and experimental results obtained in one particular furnace are shown in Fig. 2(b) to indicate the validity of this approach and the overall model. Several other circumstances were simulated, with similar results. Then the simulation results for the system can be used to predict, control and optimize the process [6].

The approach outlined above is applicable to a wide range of manufacturing systems, such as steel ingot casting, microchannel fabrication and crystal growing. In such cases, different length and time scales arise in different regions or components, making it possible to consider these separately and match the results at the boundaries.

\section{MULTIPLE SCALES WITHIN THE FLOW}

In many manufacturing processes, widely different length or time scales are encountered within the same region. The characteristics of the material being processed are determined by the transport processes that generally occur at the micro/nanoscale in the material. For instance, processes at the solid-liquid interface in crystal growing, at the surface of the heated susceptor in a CVD reactor, or at sites where chemical conversion of material occurs in food extrusion affect the quality and properties of the final product. These effects are part of the bulk flow and heat transfer. The operating conditions are imposed on the transport processes at the much larger length scales of the system. Thus, substantially different length scales arise within the domain, making it necessary to include the simulation at small scale in the large scale modeling.

Two main approaches can be employed for the numerical solution of such multiscale problems. The first approach is to solve the micro- or nanoscale problem separately and provide the results obtained as inputs to the macroscale modeling of the process. In numerical simulation, the inputs are provided at the grid points or at the control volumes for bulk flow modeling. This is the most commonly used approach. The small scales are often investigated experimentally to obtain, for instance, the rate of defect generation or of material conversion. Different experimental techniques can be used, depending on the phenomenon under consideration. For chemical conversion, for instance, the Differential Scanning Calorimeter (DSC) is used to determine the degree of conversion in biopolymers like food materials. The measured chemical kinetics as function of temperature, shear, concentration, etc., can then be entered as an input to the analytical/numerical modeling of the flow and the heat and mass transfer at discrete locations on the numerical grid. Numerical methods such as molecular dynamics can also be employed in many cases to quantify material transformation and obtain conversion or transformation rates. These results at the small scale are then incorporated in the simulation of the system. The results from the overall simulation can then be used for prediction, design and optimization of the process.

In the second approach, the simulations at the small and large length scales are carried out concurrently, rather than separately. This approach is typically more involved than the earlier one, due to changes in the model from the small to the large scale as one proceeds with the simulation. But, this approach allows greater flexibility and also results in greater accuracy in the simulation, particularly for time-dependent problems. Different grid sizes are obviously needed for the small and large scales. The small-scale modeling, using molecular dynamics, 
analytical methods, Monte Carlo or other approaches, leads to the determination of the transport properties in a control volume. Thus each control volume is first solved for the small scales and then the results obtained yield the conditions in the control volume. All the control volumes in the bulk flow are then used for the macroscopic modeling. In the examples given below, both of these approaches are applicable, though the treatment with the first approach is discussed here.

Let us consider the optical fiber manufacturing system, as sketched in Fig. 3. The system involves heating a cylindrical silica preform of diameter $10-20 \mathrm{~cm}$ and then drawing it into a fiber of diameter around $125 \mu \mathrm{m}$. The heated fiber emerging from the drawing furnace is cooled to a temperature of around $200{ }^{\circ} \mathrm{C}$ for the application of a layer of coating, which is often a polymeric material such as curable acrylates. The coating is applied in an applicator and then cured by ultra-violet radiation. Finally, the coated fiber is picked up on a spool $[7,8]$.

Thermally induced $E^{\prime}$ defects in optical fiber drawing affect the properties of the fiber. This defect is generated at a given location due to the local high temperature during the drawing process. The presence of defects causes transmission loss and reduction in mechanical strength in the fiber. Changes at the molecular level are considered to determine the generation and removal of these defects. The governing differential equation for the $E^{\prime}$ defect concentration was formulated on the basis of the thermodynamics of lattice vacancies in crystals [9]. The defects are generated due to the breaking of the $\mathrm{Si}-\mathrm{O}$ band, and these are removed by a recombination process to form $\mathrm{Si}-\mathrm{O}$ again. If the fiber is cooled very gradually after drawing, the final concentration of defects can be reduced.

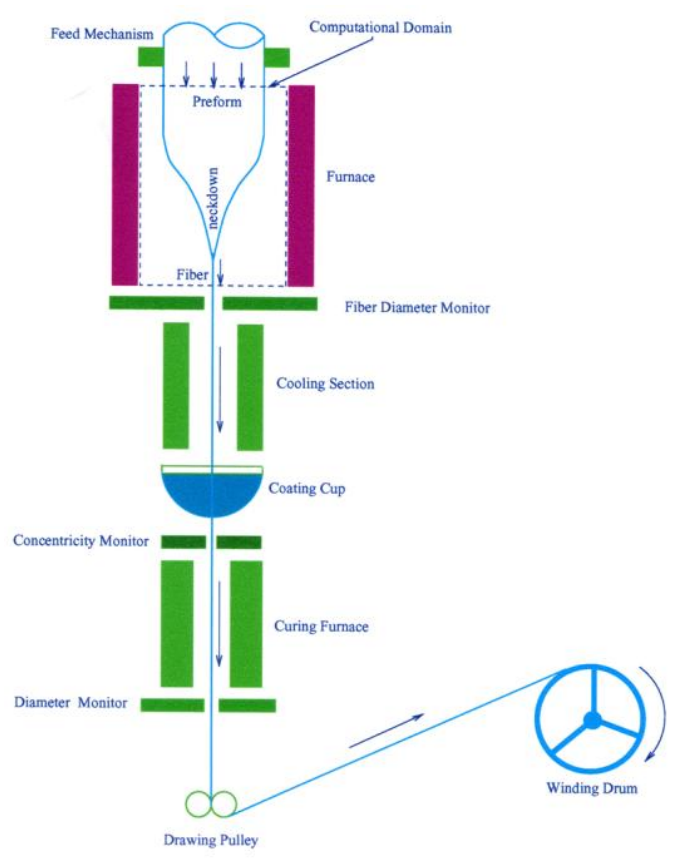

FIGURE 3. The optical fiber manufacturing system.

Thus, the net concentration of the $E^{\prime}$ defects is the difference between the generation and the recombination. The governing equation for the defect concentration is given as [9],

$$
v \frac{d n_{d}}{d z}=n_{p}(0) v^{\prime} \exp \left(-\frac{E_{p}}{K T}\right)-n_{d} v^{\prime}\left[\exp \left(-\frac{E_{p}}{K T}\right)+\exp \left(-\frac{E_{d}}{K T}\right)\right]
$$

Here, $n_{d}$ and $E_{d}$ represent the concentration and activation energy of the $E^{\prime}$ defect; while $n_{p}$ and $E_{p}$ represent those of the precursors. The initial values and constants are defined by [9]. The neck-down region in a fiber drawing process is shown in Fig. 4(a), as the fiber goes from a preform of diameter around $10 \mathrm{~cm}$ to a fiber of diameter of around $125 \mu \mathrm{m}$ [10 ]. The governing equations for the glass flow and heat transfer involve large material property changes and high viscous dissipation $\Phi$ due to the large viscosity $v$ of glass. The equations are given below for an axisymmetric flow as [10]

$$
\frac{\partial v}{\partial z}+\frac{1}{r} \frac{\partial(r u)}{\partial r}=0
$$




$$
\begin{aligned}
& \frac{\partial v}{\partial t}+u \frac{\partial v}{\partial r}+v \frac{\partial v}{\partial z}=-\frac{1}{\rho} \frac{\partial p}{\partial z}+\frac{1}{r} \frac{\partial}{\partial r}\left[r v\left(\frac{\partial v}{\partial r}+\frac{\partial u}{\partial z}\right)\right]+2 \frac{\partial}{\partial z}\left(v \frac{\partial v}{\partial z}\right) \\
& \frac{\partial u}{\partial t}+u \frac{\partial u}{\partial r}+v \frac{\partial u}{\partial z}=-\frac{1}{\rho} \frac{\partial p}{\partial r}+\frac{2}{r} \frac{\partial}{\partial r}\left(r v \frac{\partial u}{\partial r}\right)+\frac{\partial}{\partial z}\left[v\left(\frac{\partial v}{\partial r}+\frac{\partial u}{\partial z}\right)\right]-\frac{2 v u}{r^{2}}
\end{aligned}
$$

$\rho C_{p}\left(\frac{\partial T}{\partial t}+u \frac{\partial T}{\partial r}+v \frac{\partial T}{\partial z}\right)=\frac{1}{r} \frac{\partial}{\partial r}\left(r K \frac{\partial T}{\partial r}\right)+\frac{\partial}{\partial z}\left(K \frac{\partial T}{\partial z}\right)+\Phi+S_{r}$



(a)

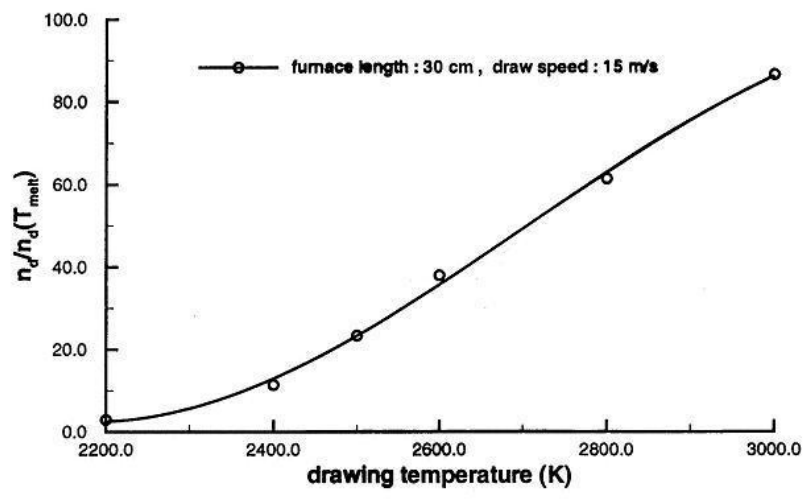

(b)

FIGURE 4: (a) Neck-down region in optical fiber drawing; (b) Average concentration of $E$ ' defects as a function of the furnace wall temperature;
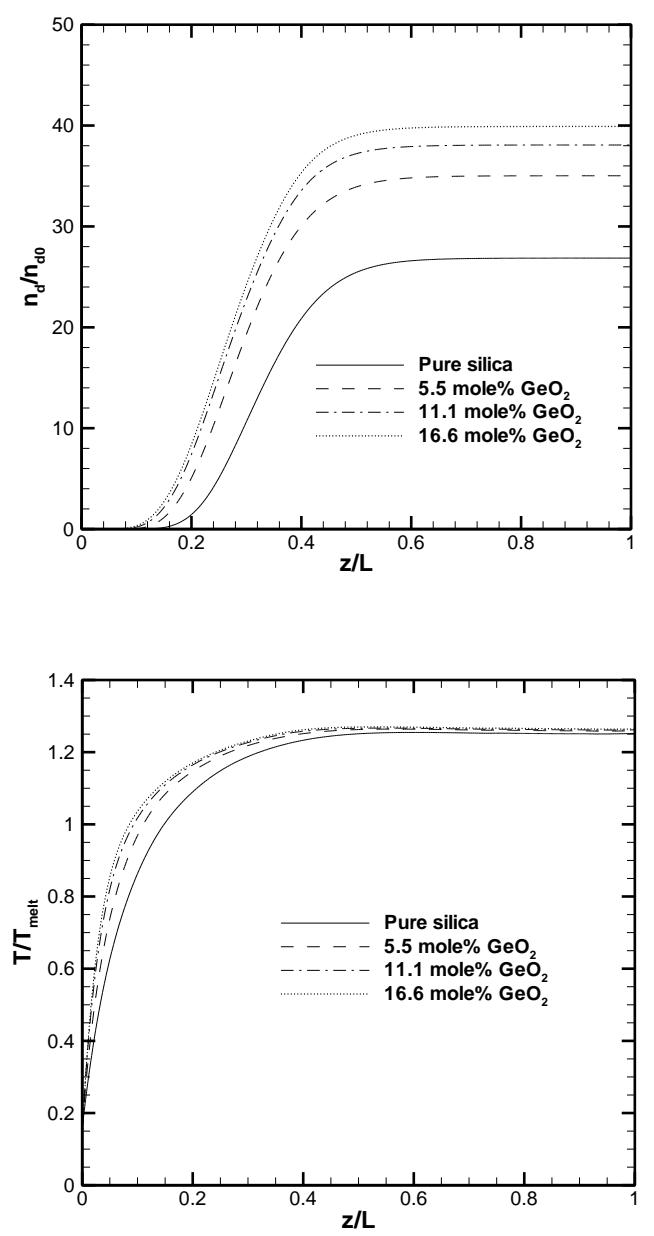

FIGURE 5. (a) Variation of the concentration of $E^{\prime}$ defects along the centerline for various $\mathrm{GeO}_{2}$ concentrations in a doped fiber; (b) Temperature distribution in a doped fiber.

The variation of the average concentration of $E^{\prime}$ defects with the drawing temperature is shown in Fig. 4(b). It is clearly seen that the temperature is critical to the generation of these defects and an increase in temperature gives rise to a larger concentration of defects due to the higher breakage of the $\mathrm{Si}-\mathrm{O}$ bond. In various specialty fibers for diagnostics, biomedical systems and other applications, dopants, such as rare-earth elements, are generally added to the preform to vary the characteristics of the optical fiber. The drawing of doped fibers may be simulated in a manner similar to that for pure fibers [11]. The defects in a doped fiber are shown in Fig. 5 (a) and the temperature distribution in Fig. 5 (b). Clearly, the effect can be controlled by doping and by varying the operating conditions, particularly the furnace temperature. Again, these effects arise due to the microscopic mechanisms 
operating at the level of the defects and the dopants. The final product is controlled by the operating conditions like preform and fiber speeds, preform diameter, furnace temperature, and coating fluid temperature and pressure. The geometry and dimensions of the system also affect the fiber characteristics.

Another important manufacturing process is the extrusion of plastics, food and other polymers, as shown in Fig. 1(c). This process is critical to the food industry, as well as to plastics. As in the case of optical fiber drawing, the microsopic changes in the material being extruded depend on the operating conditions, such as temperature and RPM, which are imposed at the engineering scale, as well as dimensions and geometry of the system components. The material transformation process is represented by the chemical kinetics, which is dependent on the temperature, shear and concentration [12]. Obviously, the micro- and nanoscale conversion mechanisms must be coupled with the polymer flow and heat transfer to determine the degree of conversion, pressure rise in the extruder, bulk temperature and other important quantities. Figure 6(a) qualitatively shows some of the conversion mechanisms for food. The chemical kinetics can be determined experimentally and provided as input to the model of the flow at each location. Figure 6(b) shows the sketch of a particular extrusion die and the corresponding simulation and experimental results. The good agreement between the two lends support to the experimentally determined chemical kinetics and the predictability of the overall model. Several other similar studies were carried out to validate the models by comparisons of degree of conversion, temperature and pressure with experimental data [13].

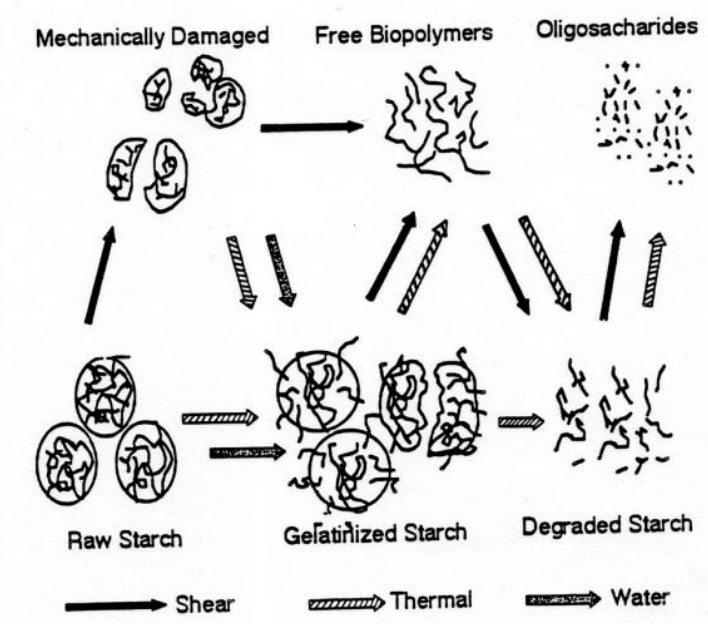

(a)

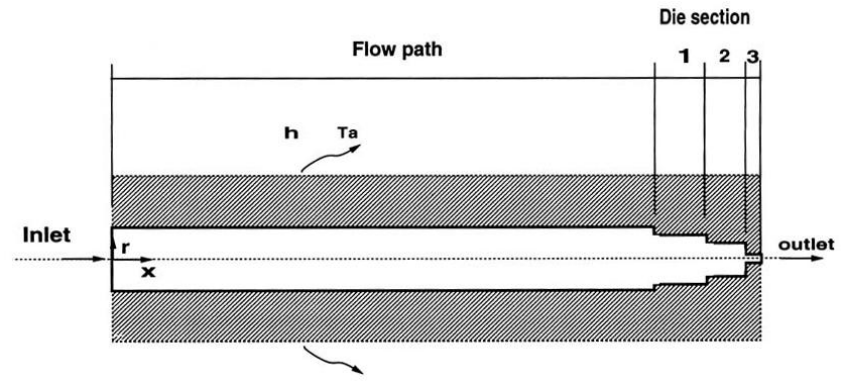

Table 1. Comparison Between Predicted and Experimental Results on the Pressure Drop for the Flow of Corn Meal in a Food Extrusion Die. Case 1: $m_{c}=21 \%, T_{i n}=382 \mathrm{~K}, \dot{m}=$ $51.8 \mathrm{~kg} / \mathrm{h}$. Case 2: $m_{c}=17.3 \%, T_{i n}=394 K, \dot{m}=59.4 \mathrm{~kg} / \mathrm{h}$.

\begin{tabular}{cccr}
\hline Case & $\begin{array}{c}\text { Simulation } \\
\Delta \mathbf{p}(\mathrm{MPa})\end{array}$ & $\begin{array}{c}\text { Experiment } \\
\Delta \mathbf{p}(\mathrm{MPa})\end{array}$ & Error \\
\hline 1 & 6.275 & 6.611 & $5.35 \%$ \\
2 & 6.204 & 6.263 & $1.02 \%$ \\
\hline
\end{tabular}

(b)

Figure 6: (a) Basic chemical conversion driven by shear and temperature in pure starch; (b) Sketch of an extrusion die and the comparison between calculated and experimentally obtained pressure drop in the die.

Chemical kinetics plays a critical role in the deposition of material from the gas phase in chemical vapor deposition systems as well $[14,15]$. The concentrations of the various chemical species in the reactor affect the chemical kinetics, which in turn affects the film deposition. In many cases, the process is limited by the chemical kinetics, implying that the transport processes are quite vigorous and the deposition is restricted largely by the kinetics. The chemical kinetics for several important processes for material deposition is available in the literature. But this is often complicated by the large number of reactions and species involved. Also, reactions at the surface, as well as within the gas, need to be simulated. If we consider Silicon deposition from Silane $\left(\mathrm{SiH}_{4}\right)$ with Hydrogen as the carrier gas in a CVD reactor, the chemical kinetics for the deposition can be approximated by the following simple expression [16]

$\mathrm{K}=\frac{K_{o} p_{S i H 4}}{1+K_{1} p_{H 2}+K_{2} p_{S i H 4}}$

Here, $\mathrm{K}$ is the surface reaction rate in mole of $\mathrm{Si} / \mathrm{m}^{2} \mathrm{~s}, \mathrm{~K}_{\mathrm{O}}=$ $\mathrm{A} \exp (-\mathrm{E} / \mathrm{RT}), \mathrm{E}$ being the activation energy, and $\mathrm{A}, \mathrm{K}_{1}$, and $\mathrm{K}_{2}$ are constants which are obtained experimentally. The p's are the partial pressures of the two species in the reactor. 


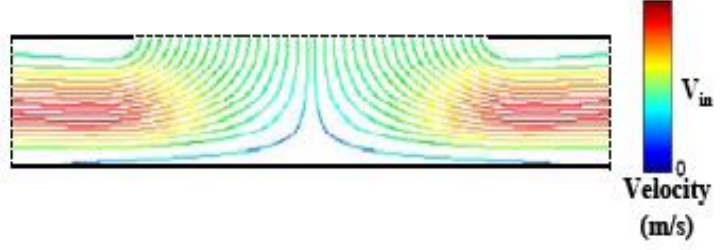

(a)



(K)

(b)

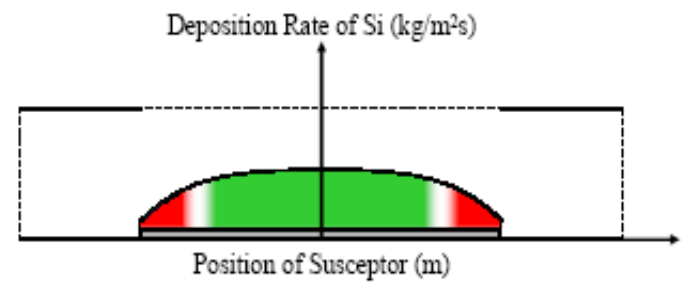

(c)

Figure 7. (a) Calculated streamlines in an impingement CVD reactor; (b) Isotherms in the flow; (c) Typical thin film deposited, showing non-uniformity in thickness.

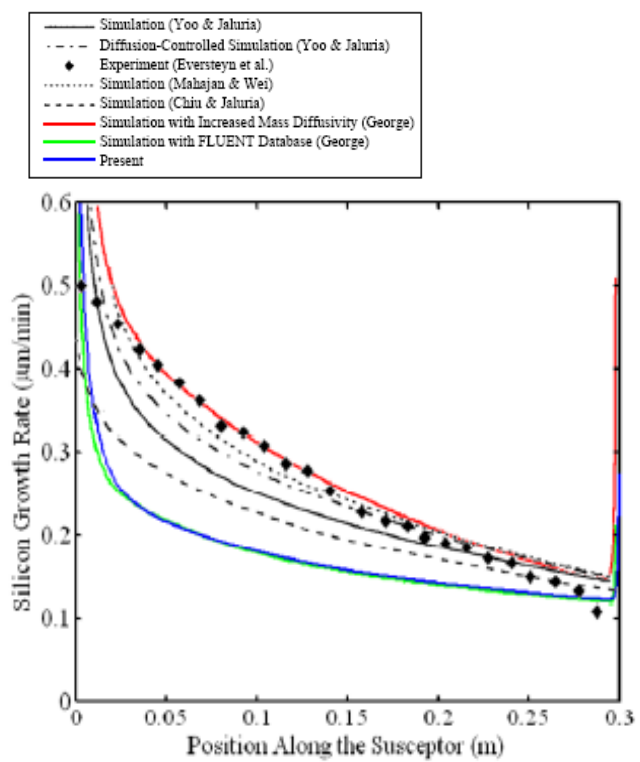

(d)

FIGURE 8. Comparison between numerical predictions and experimental results for $\mathrm{Si}$ deposition for a variety of simulations.
Some typical results on the transport and deposition of Silicon from Silane are shown in Fig. 7 for an impingement type CVD [17]. Important considerations are the nonuniformity of the deposited film and the presence of defects in the film. Characterization studies are carried out to ensure that the properties of the thin film are acceptable for the applications of interest. Also, extensive efforts are made to achieve better uniformity and film quality by suitable systems design and by controlling the operating conditions like susceptor temperature and inflow velocity and concentration. Substantial work has been done on this process because of its wide applicability in material fabrication. A comparison with experimental results is shown in Fig. 8 for Silicon deposition for model validation. Deposition of several other materials like TiN, SiC, GaN and GaAs have been investigated for a range of applications in electronic and optical systems [18]. Thus, this brief discussion, along with the examples, illustrate the approach for modelling such multiscale problems, which involve both micro- and macroscale mechanisms in the system.

\section{CONCLUSIONS}

This paper discusses the mathematical and numerical modeling of multiscale problems in the thermal processing of materials for manufacturing. In most cases, the thermal process and the system involve significantly different length and time scales over different components or regions. It is important to obtain accurate simulation results to predict the system behaviour and to design and optimize the process. Two important circumstances are considered in detail. The first arises when the different scales occur in different, separate, regions. Then, the different regions can be investigated separately by employing the relevant mechanisms and experimentation. The results obtained in different regions are then matched at the boundaries, or the results from the smaller scale provide the boundary conditions for the larger scale domain. Reduced order models may be used in some problems for some components to simplify the simulation. Large differences in time scales are handled in a similar manner. Examples are used to illustrate this circumstance and to present characteristic results.

The second circumstance is the one in which multiple length or time scales arise in the same domain. The basic approach of solving the micro- or nanoscale problem separately by analysis or experimentation and then providing the results as inputs to the macroscopic model of the system is discussed. Analytical/numerical modelling, as well as experimentation, may be used to provide the needed inputs. Typical results are presented and discussed. 
It is important to link the simulation at different scales to obtain results that can form the basis for system control and optimization to obtain high quality products at low cost. Some of the relevant considerations in this important area are briefly presented in this paper.

\section{ACKNOWLEDGMENTS}

The author acknowledges the partial support from NSF under grant number 1067141, and from industry for this study and the work done by several graduate students.

\section{REFERENCES}

[1] Jaluria, Y., 2003. "Thermal processing of materials: From basic research to engineering". ASME J. Heat Transfer, 125, pp. 957-979.

[2] Mahajan, R.L., 1996. "Transport phenomena in chemical vapor-deposition systems". Advances in Heat Transfer, 28, pp. 339-425.

[3] Jaluria, Y., 2009. "Microscale transport phenomena in materials processing”. ASME J. Heat Transfer, 131, pp. 033111-1-17.

[4] Jaluria, Y., 1988. "Numerical simulation of the transport processes in a heat treatment furnace". Int. J. Num. Meth. Engg., 25, pp. 387-399.

[5] Joshi, Y., 2012. "Reduced order thermal models of multiscale microsystems". ASME J. Heat Transfer, 134, pp. 031008-1-11.

[6] Jaluria, Y., 2008. Design and Optimization of Thermal Systems, Second Edition, CRC Press (Taylor \& Francis), Boca Raton, FL.

[7] Paek, U.C., 1999. "Free drawing and polymer coating of silica glass optical fibers". ASME Journal of Heat transfer, 121, pp. 774-788.

[8] Roy Choudhury, S. and Jaluria, Y., 1998, "Practical aspects in the drawing of an optical fiber." J. Mater. Res., 13, pp. 483-493.

[9] Hanafusa, H., Hibino, Y., Yamamoto, F., 1985. "Formation mechanism of drawing-induced E' centers in silica optical fibers”. J. Appl. Phys., 58(3), pp. 1356-1361.

[10] Cheng, X. and Jaluria, Y., 2005. "Effect of furnace thermal configuration on optical fiber heating and drawing." Numerical Heat Transfer, 48, pp. 507528.

[11] Chen, C. and Jaluria, Y., 2009. "Effects of doping on the optical fiber drawing process". Int. J. Heat Mass Transfer, 52, pp. 4812-4822.

[12] Wang, S.S., Chiang, C.C., Yeh, A.I., Zhao, B. and Kim, I.H., 1989. "Kinetics of phase transition of waxy corn starch at extrusion temperatures and moisture contents, J. Food Sci., 54, pp. 1298-1301.

[13] Zhu, W. and Jaluria, Y., 2001. "Residence time and conversion in the extrusion of chemically reactive materials." Polymer Engg. Sci., 41, pp. 1280-1291.

[14] Jensen, K.F., Einset, E.O. and Fotiadis, D.I., 1991. "Flow phenomena in chemical vapor deposition of thin films". Ann. Rev. Fluid Mechanics, 23, pp. 197232.

[15] Wu, B., Ma, R. and Zhang, H., 2003. "Epitaxy growth kinetics of GaN films." Journal of Crystal Growth, 250(1-2), pp. 14-21.

[16] Eversteyn, F.C., Severin, P.J.W., Brekel, C.H.J. and Peek, H.L., 1970. "A stagnant layer model for the epitaxial growth of silicon from silane in a horizontal reactor". J. Electrochem. Soc., 117, pp. 925-931.

[17] Lin, Po-Ting, Gea, H.C. and Jaluria, Y., 2009. "Parametric modeling and optimization of chemical vapor deposition process." J. Manuf. Sci. Eng., 131, pp. 011011-1 to 7.

[18] Meng, J. and Jaluria, Y., 2013. "Numerical simulation of $\mathrm{GaN}$ growth in a metalorganic chemical vapor deposition process". J. Mfg. Sci. Eng., 135, pp. 061013-1 to 7. 
Main Gas Flow

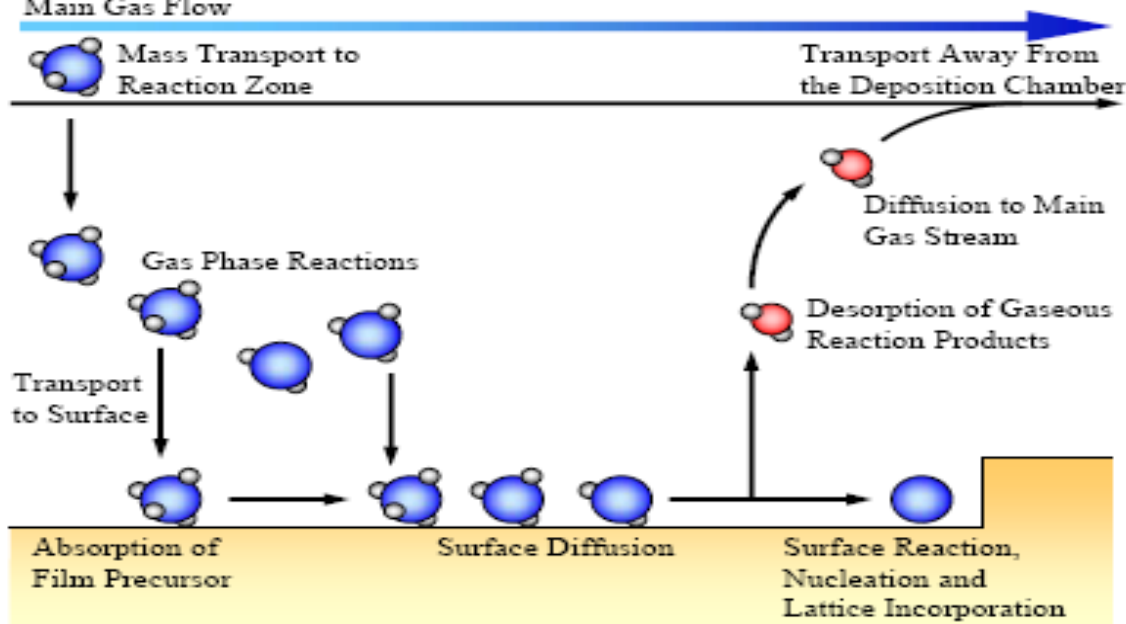

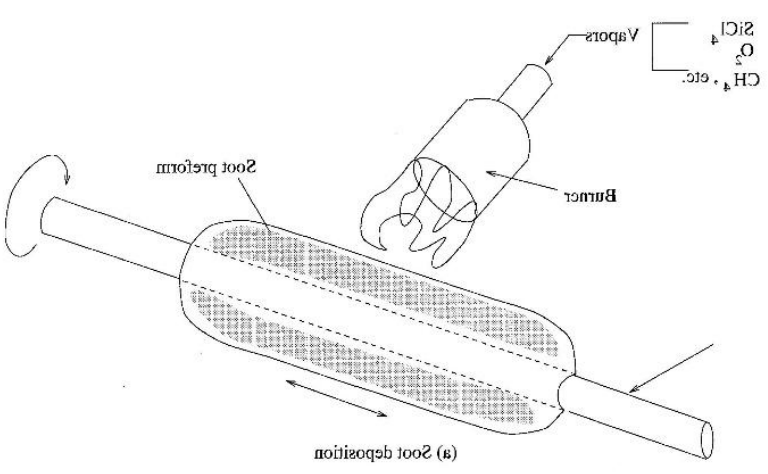

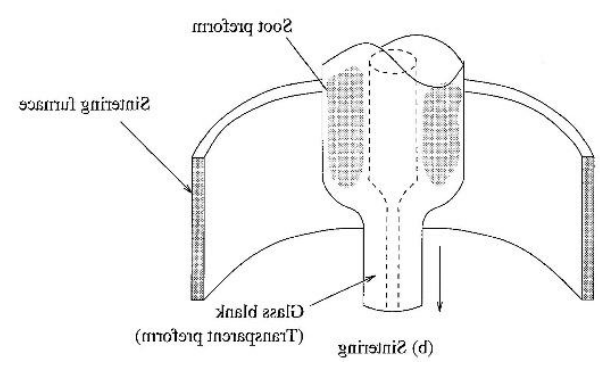

Chemical Vapor Deposition (CVD) for thin films and Outer Vapor Deposition (OVD) for fabrication of optical fiber preforms: Chemical Transformations and Deposition at Micro/Nanometer Scale and System at Macro or Engineering Scale 